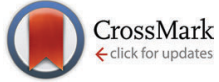

Cite this: J. Mater. Chem. C, 2015, 3, 8942

Received 25th June 2015, Accepted 1st August 2015

DOI: $10.1039 / \mathrm{c} 5 \mathrm{tc} 01888 \mathrm{a}$

www.rsc.org/MaterialsC

\title{
Cholinium-based ion gels as solid electrolytes for long-term cutaneous electrophysiology $\dagger$
}

\author{
Mehmet Isik, $\ddagger^{\mathrm{a}}$ Thomas Lonjaret, $\ddagger^{\mathrm{bc}}$ Haritz Sardon, ${ }^{\mathrm{a}}$ Rebeca Marcilla, ${ }^{\mathrm{d}}$ \\ Thierry Herve, ${ }^{c}$ George G. Malliaras, ${ }^{b}$ Esma Ismailova*b ${ }^{*}$ and David Mecerreyes*ae
}

\begin{abstract}
Cholinium-based bio-ion gels were prepared by photopolymerization of poly(cholinium lactate methacrylate) network within cholinium lactate ionic liquid. The rheological and thermal properties as well as ionic conductivity of ion gels of different compositions were measured. As indicated by rheological measurements, the ion gels show the properties of gel materials which become soft by increasing the amount of free ionic liquid. Cholinium ion gels with various composition of free ionic liquid vs. methacrylic network show glass transitions between $-40^{\circ}$ and $-70{ }^{\circ} \mathrm{C}$ and thermal stability up to $200{ }^{\circ} \mathrm{C}$. The ionic conductivity of these gels increases from $10^{-8}$ to $10^{-3} \mathrm{~S} \mathrm{~cm}^{-1}$ at $20{ }^{\circ} \mathrm{C}$ by varying the amount of free ionic liquid between 0 and $60 \mathrm{wt} \%$, respectively. Low glass transition temperature and enhanced ionic conductivity make the cholinium-based ion gels good candidates to be used as a solid electrolytic interface between the skin and an electrode. The ion gels decrease the impedance with the human skin to levels that are similar to commercial $\mathrm{Ag} / \mathrm{AgCl}$ electrodes. Accurate physiologic signals such as electrocardiography (ECG) were recorded with ion gels assisted electrodes for a long period of time (up to $72 \mathrm{~h}$ ) with a remarkable stability. The low toxicity and superior ambient stability of cholinium ionic liquids and ion gels make these materials highly attractive for long-term cutaneous electrophysiology and other biomedical applications.
\end{abstract}

\section{Introduction}

Electroencephalography (EEG) (brain activity), electrocardiography (ECG) (cardiac activity) or electromyography (muscular response) are important electrophysiological diagnostic procedures where electrodes placed on the skin are used to monitor the electrical activity of specific organs. Ag/AgCl electrodes are the current gold standard in cutaneous recordings but they require the use of an electrolyte to decrease electrode/skin interface impedance. When exposed to air, the electrolyte dries out after several hours causing an increase in the impedance resulting in the loss of accurate recording. ${ }^{1}$ Further addition or refilling of the electrolyte gel to

${ }^{a}$ POLYMAT, University of the Basque Country UPV/EHU, Avda. Tolosa 72, 20018, San Sebastian, Spain. E-mail: david.mecerreyes@ehu.es; Fax: +34 943 506062; Tel: +34 943018018

${ }^{b}$ Department of Bioelectronics Centre Microélectronique de Provence Ecole Nationale Supérieure des Mines de Saint Etienne 880 route de Mimet, 13541 Gardanne, France. E-mail: ismailova@emse.fr

${ }^{c}$ Microvitae Technologies, Hôtel Technologique, Europarc Sainte Victoire Bât 6, Route de Valbrillant, 13590 Meyreuil, France

${ }^{d}$ Electrochemical Processes Unit, IMDEA Energy Institute, Parque Tecnológico de Móstoles, Avda. Ramón de la Sagra 3, 28935 Móstoles, Spain

${ }^{e}$ IKERBASQUE, Basque Foundation for Science, E-48011, Bilbao, Spain

$\dagger$ Electronic supplementary information (ESI) available: ${ }^{1} \mathrm{H}$ NMR, rheology, ATRFTIR, DSC, TGA, moisture uptake. See DOI: 10.1039/c5tc01888a

\$ These authors contribute equally to this paper. each electrode is time-consuming and causes discomfort to the patient and the caregiver. All these factors reveal that currently used cutaneous electrodes present shortcomings for long-term recordings. Hence, there is a thriving interest to develop new electrolyte systems with low evaporation rates to be used in electrodes aimed for long-term cutaneous recordings.

Ionic liquids (ILs) are ionic compounds with a negligible vapor pressure and evaporation rate. ${ }^{2}$ Such liquids integrated in a gel matrix allow to overcome ambient stability limitations of the actual electrolyte systems used for electrophysiological recordings. ${ }^{3}$ Recently, the potential of an ion gel or ionic liquid gel assisted PEDOT:PSS electrodes has been successfully demonstrated in cutaneous applications underlying their high performance compared to commercial electrodes with an aqueous-based gel. ${ }^{3}$ The reported ion gel was based on 1-ethyl-3methylimidazolium ethyl sulfate ionic liquid formulated within a poly(ethylene glycol) diacrylate network.

Although ionic liquids were initially defined as green solvents, one of the most actual concerns regarding ionic liquids is their toxicology. ${ }^{4}$ It is widely accepted that among all the different types of ionic liquids some of them are toxic and hence should not be released to the environment while some others not. ${ }^{5}$ As a general rule even though there is a certain contribution from the anion, ${ }^{6}$ the toxicity of the ionic liquid is largely determined by the headgroup of the cation. ${ }^{7-9}$ Cholinium refers to trimethylethanol 
ammonium cation and it is an essential micronutrient assisting several biological functions. For this reason, cholinium cationbased ionic liquids are known also as bioionic liquids due to their biocompatibility, biodegradability and low toxicity. ${ }^{10,11}$

In this article, we report the preparation and the characterization of cholinium-based ion gels and their use as solid electrolytes in cutaneous electrophysiology. Ionic liquids are usually immobilized in gels to provide them solid-like mechanical properties while preserving their unique ionic conductivity. ${ }^{12,13}$ Ion gels are already introduced as solid electrolytes in different devices such as batteries and supercapacitors, ${ }^{14}$ fuel cells, ${ }^{15}$ electrochemical and biochemical sensors, ${ }^{16}$ actuators, ${ }^{17}$ field-effect transistors ${ }^{18,19}$ or drug delivery systems. ${ }^{20}$ In our work, we propose to incorporate cholinium ion gels to assist surface electrodes in cutaneous electrophysiology aimed to be performed during long time. Ion gels were obtained by confining the ionic liquids into a covalently cross-linked poly(ionic liquid) network prepared by photopolymerization. We first studied thermal, electrical and rheological properties of the ion gels by varying the amount of free cholinium lactate ionic liquid in the polymeric matrix. Then, these ion gels were deposited onto PEDOT:PSS-based electrodes and we investigated their long-term recording abilities from the skin.

\section{Results and discussion}

\section{Preparation of cholinium-based ion gels}

Ion gels are a particular type of gel electrolytes where the role of solvent (as water in hydrogels) is played by nonflammable and nonvolatile ionic liquids. It is worth to note that ion gels can be prepared using different types of polymeric matrices such as cellulose, poly(ethylene oxide) or poly(ionic liquid)s or even inorganic siloxane networks made by sol-gel chemistry. ${ }^{13}$ However, poly(ionic liquid) networks are usually preferred due to their excellent compatibility with the free ionic liquids preventing their future leaching problems. In this work, ion gels were prepared through photopolymerization of the ionic liquid monomer (cholinium lactate methacrylate) with the ionic liquid (cholinium lactate) and a small amount (1-2\%) of a commercially available crosslinker such as ethylene glycol dimethacrylate. The amount of free IL with respect to the polymer matrix was varied from $0 \mathrm{wt} \%$ to $60 \mathrm{wt} \%$. Scheme 1 shows a representation of the preparation method of the ion gels. The ion gels were coded as cholinium IG-0, IG-10, IG-20, IG-30, IG-40, IG-50 and IG-60 where the number represents the weight percent of the free ionic liquid.

Photopolymerization is a common technique to produce polymers at room temperature and short reaction times. Therefore, all the ion gels in this study were prepared in less than 2 minutes using a UV light-curing conveyor-belt system. One of the important parameters regarding the synthetic reproducibility and proper design of ion gels through photopolymerization is the conversion of the monomer to form the polymer matrix. Uncontrolled polymerization will yield non-reproducible ion gels which will result in gels with incoherent properties. The conversion of the polymerization was controlled by ${ }^{1} \mathrm{H}$ NMR and ATR-FTIR spectroscopy. The crosslinked structures of ion gels prevent their

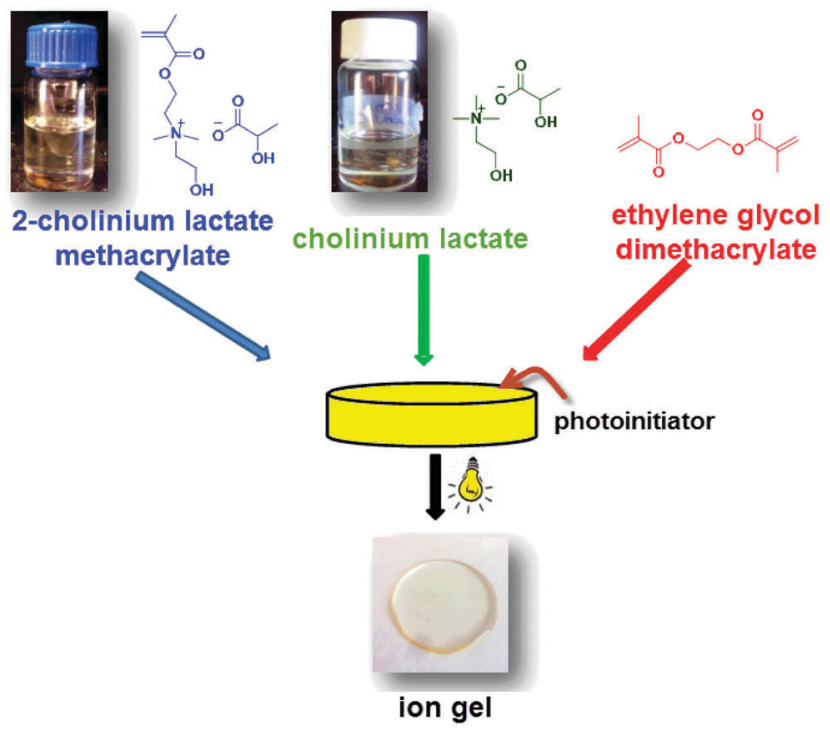

Scheme 1 General pathway for the preparation of cholinium lactate ion gels.

dissolution and make it impossible to obtain direct ${ }^{1} \mathrm{H}$ NMR spectra from solution. Therefore, to evaluate non-polymerized content of the monomer, the ion gels were immersed into $\mathrm{D}_{2} \mathrm{O}$ for 1 day. The soluble fraction was then analyzed by ${ }^{1} \mathrm{H}$ NMR spectroscopy. Since the cholinium lactate methacrylate ionic liquid monomer is highly soluble in water, any unreacted monomer will be dissolved in the aqueous phase. As it can be seen from Fig. S1 (ESI $\dagger$ ), the signals associated with the reactive methacrylic group disappeared for all the ion gel compositions after exposure to UV light indicating the complete photopolymerization of the monomer. The further studies using ATR-FTIR spectroscopy affirm the full conversion of the monomer in solid ion gel samples. Fig. S2 (ESI $\dagger$ ) shows the absence of methacrylic stretching band associated with the ionic liquid monomer. This further proves the successful polymerization of the ionic liquid monomer to form the polymer matrix.

\section{Rheological properties of the ion gels}

The mechanical properties of ion gels are one of the most important properties for their practical application as part of the electrode in contact with the skin. This interface has to be soft to conform with skin surface and hair, flexible to absorb mechanical shocks. Therefore, rheological properties of the ion gels were analyzed by frequency sweep measurements. Elastic $\left(G^{\prime}\right)$ and viscous $\left(G^{\prime \prime}\right)$ moduli of the ion gels were examined as a function of angular frequency at $25{ }^{\circ} \mathrm{C}$ as shown in Fig. 1 for 3 different ion gel formulations.

Ideal gels display almost purely elastic response where the elastic modulus is higher than the viscous modulus and is independent of angular frequency. For the sample that does not contain any free IL (IG-0), highest elastic modulus $\left(G^{\prime}\right)$ was obtained which is around 4.08 $\mathrm{MPa}$ and is approximately four times greater than the loss modulus $\left(G^{\prime \prime}\right)$ throughout the entire frequency range. Both $G^{\prime}$ and $G^{\prime \prime}$ were frequency independent until $1 \mathrm{rad} \mathrm{s}^{-1}$ and after there was a very weak dependency. This 


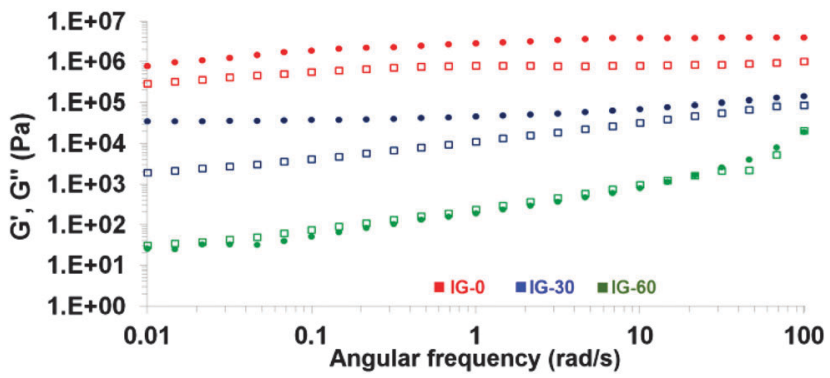

Fig. 1 Frequency dependences of dynamic storage $G^{\prime}$ (circular symbols) and loss $G^{\prime \prime}$ moduli ( $\square$ square symbols) of the ion gels.

confirms that the sample was a gel network of rubbery nature. As expected, introduction of free ionic liquid into the polymer network acts as plasticizer and reduces the mechanical strength of the ion gels as revealed by the decreased $G^{\prime}$ and $G^{\prime \prime}$ values. With the introduction of $10 \%$ IL into the structure, the $G^{\prime}$ value decreased to $0.83 \mathrm{MPa}$ at $100 \mathrm{rad} \mathrm{s}^{-1}$ (Fig. S3, ESI $\dagger$ ). The $G^{\prime \prime}$ was lower than $G^{\prime}$ over the entire angular frequency range and both moduli were slightly frequency dependent indicating that the material behaves as a soft solid material. Although increasing the IL content further reduced the elastic modulus down to $0.55 \mathrm{MPa}, G^{\prime \prime}$ was lower than the $G^{\prime}$ over the entire angular frequency range and dependence of both moduli was similar to the previous formulation indicating a soft solid ion gel. The gradual decrease of both moduli was observed while increasing free IL content in the ion gel formulations. All the ion gels until $50 \% \mathrm{IL}$ displayed similar frequency behaviour over the entire measured angular frequency range. In all cases, the elastic moduli of the ion gels were higher than loss moduli suggesting that the elastic components of the dynamic moduli were greater. The ion gel IG-60 is the sample that contains the highest amount of free IL and displayed the lowest mechanical property relative to the samples containing less IL. At high angular frequencies, the $G^{\prime}$ and $G^{\prime \prime}$ values were almost the same around $0.02 \mathrm{MPa}$. The $G^{\prime \prime}$ became higher than $G^{\prime}$ over the entire lower angular frequency range and both moduli were frequency dependent indicating that the sample was a pregel. These findings indicated the gel type behaviour of the ion gels due to the crosslinked poly(ionic liquid) matrix blended with ionic liquid. All in all, these cholinium ion gels presented soft solid and elastic behaviours which are ideal mechanical properties for the application as cutaneous electrodes.

\section{Thermal properties of the ion gels}

Thermal properties of the ion gels were analyzed by differential scanning calorimetry (DSC) and thermogravimetric analysis (TGA). DSC signals are provided in Fig. 2a.

The glass transition temperature $\left(T_{\mathrm{g}}\right)$ of the homopolymer of 2-cholinium lactate methacrylate is around $-50{ }^{\circ} \mathrm{C} .{ }^{21}$ Similarly, DSC analysis of the sample IG-0 revealed a $T_{\mathrm{g}}$ around $-40{ }^{\circ} \mathrm{C}$ which is slightly higher due to the crosslinked nature of the sample. Introduction of free IL acts as a plasticizer to the polymer matrix decreasing the $T_{\mathrm{g}}$ of the ion gel. Therefore, $T_{\mathrm{g}}$ values gradually decrease with the increasing IL content. The
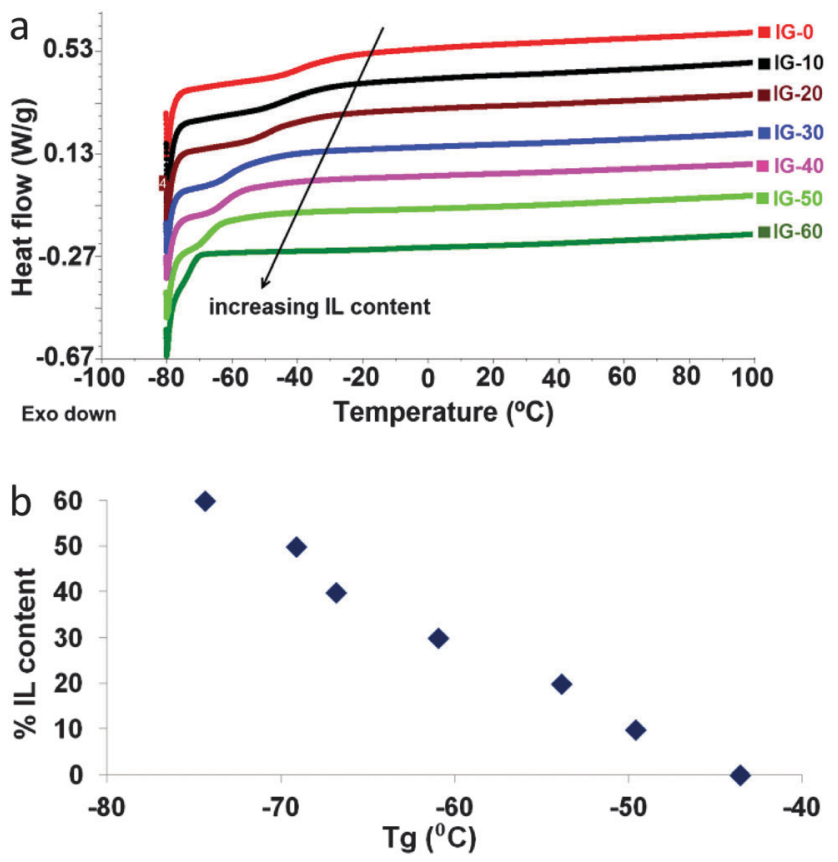

Fig. 2 (a) Differential scanning calorimetry (DSC) curves of the ion gels containing different amounts of IL. (b) Glass transition temperature change vs. free ionic liquid \%.

lowest glass transition being found around $-70{ }^{\circ} \mathrm{C}$ for IG-60 in which $60 \%$ free IL content was blended. Additionally, the change in the glass transition temperature with changing ionic liquid content is provided in Fig. 2 b. As clearly seen from the figure, addition of ionic liquid plasticizes the material resulting in a gradual decrease of the glass transition temperature with increasing amount of free ionic liquid in the polymeric matrix. Low $T_{\mathrm{g}}$ behaviour of ion gels provides a broad operational window from $-70{ }^{\circ} \mathrm{C}$ to higher temperatures.

To determine the thermal stability of ion gels, TGA measurements were carried out. The TGA signals are provided in Fig. 3 a. Additional TGA curves focusing in $30-100{ }^{\circ} \mathrm{C}$ temperature range are also given in Fig. S4 (ESI + ). As reported in literature, cholinium based ionic liquids display onset temperatures starting from $170{ }^{\circ} \mathrm{C} .{ }^{11}$ For the ion gels composed of cholinium cation combined with lactate anion, the trend observed for the thermal stabilities were similar to the glass transition temperatures. Although the onset temperatures were similar to each other for all the ion gel formulations at around $180{ }^{\circ} \mathrm{C}$, the weight loss after that point were distinctive as it can be seen from Fig. 3. Samples containing higher amounts of free IL displayed a sharper weight loss compared to the samples with low IL contents. The sample IG-0 showed a decomposition temperature $\left(50 \%\right.$ weight loss) at $292{ }^{\circ} \mathrm{C}$ and this temperature gradually dropped down to $250{ }^{\circ} \mathrm{C}$ for IG-60. To better demonstrate the change in the decomposition temperature of the ion gels with changing ionic liquid content, Fig. $3 \mathrm{~b}$ is provided. As clearly seen, introduction of ionic liquid into the solid matrix gradually decreases the decomposition temperature as the amount of ionic liquid increases.

To summarize, the ion gels showed in general glass transition temperatures way below room temperature and good 
a
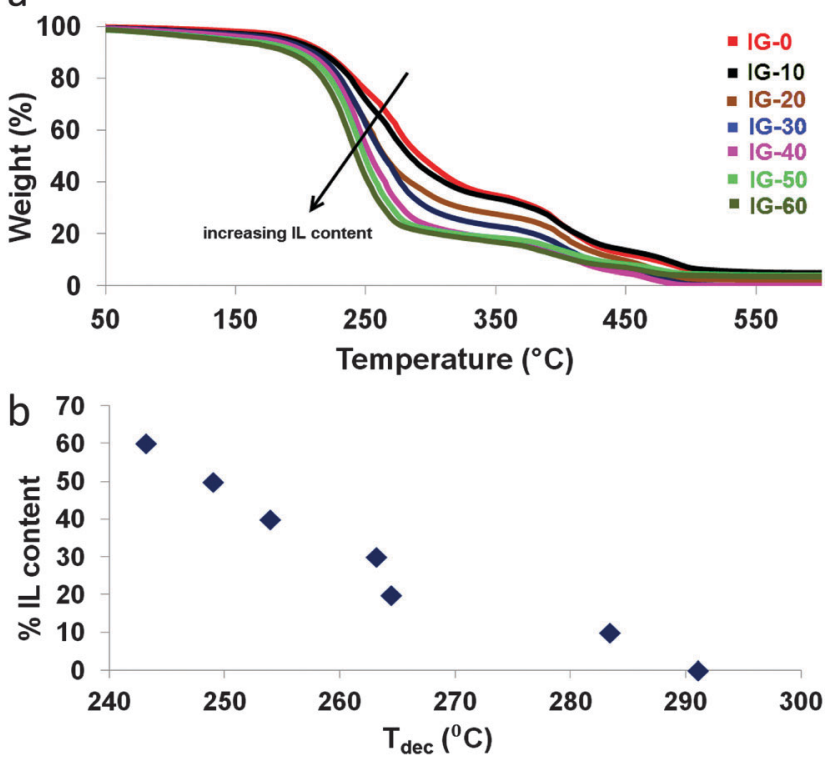

Fig. 3 (a) Thermal gravimetric analysis (TGA) profiles of the cholinium ion gels. (b) Decomposition temperature vs. free IL content in the gels (the temperature at which $50 \%$ weight loss was observed and was taken as the decomposition temperature).

thermal stability up to $200{ }^{\circ} \mathrm{C}$ which provides a flexible operational window.

\section{Electrical properties of the ion gels}

Due to the presence of the free IL embedded in the polymer matrix, ion gels present higher ionic conductivities than other solid polymer electrolytes. ${ }^{14 b}$ Therefore, the ionic conductivity of an ion gel becomes one of the most characteristic properties and it is usually related to its good performance in the different device application. For this reason, the ionic conductivities of ion gels were determined by impedance spectroscopy from $0{ }^{\circ} \mathrm{C}$ to $110{ }^{\circ} \mathrm{C}$ as shown in Fig. 4 .

The ionic conductivities change dramatically with the introduction of free ionic liquid and its weight content as expected. The slight curvature observed in the Arrhenius plot is in good accordance with the Vogel-Tamman-Fulcher (VTF) model which is commonly seen in amorphous polymer electrolytes

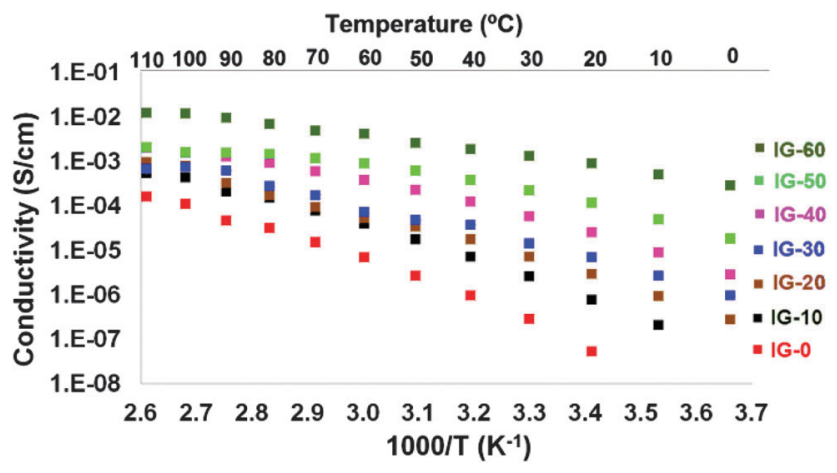

Fig. 4 lonic conductivities of the ion gels from $0{ }^{\circ} \mathrm{C}$ to $110{ }^{\circ} \mathrm{C}$. due to the amorphous nature of the polymer matrix. The conductivity values were gradually increasing with the increasing free ionic liquid amount in the ion gel. The ionic conductivity reach values between $10^{-8} \mathrm{~S} \mathrm{~cm}^{-1}$ for the pure poly(ionic liquid) and $10^{-3} \mathrm{~S} \mathrm{~cm}^{-1}$ for ion gels having $60 \mathrm{wt} \%$ of free IL. The values range from $10^{-8} \mathrm{~S} \mathrm{~cm}^{-1}$ at $20^{\circ} \mathrm{C}$ to $10^{-4} \mathrm{~S} \mathrm{~cm}^{-1}$ at $110{ }^{\circ} \mathrm{C}$ for the sample that does not contain any free IL. When $20 \mathrm{wt} \%$ of cholinium lactate is introduced into the ion gel, the ionic conductivity at $20{ }^{\circ} \mathrm{C}$ was $10^{-6} \mathrm{~S} \mathrm{~cm}^{-1}$ which gradually increased to $10^{-3} \mathrm{~S} \mathrm{~cm}^{-1}$ at $110{ }^{\circ} \mathrm{C}$. As expected, the highest ionic conductivity values were obtained for the ion gel which contains $60 \mathrm{wt} \%$ free ionic liquid IG-60, the values ranging from $10^{-3} \mathrm{~S} \mathrm{~cm}^{-1}$ at $20{ }^{\circ} \mathrm{C}$ to $10^{-2} \mathrm{~S} \mathrm{~cm}^{-1}$ from $20{ }^{\circ} \mathrm{C}$ to $110{ }^{\circ} \mathrm{C}$, respectively.

\section{Application of ion gels as electrolyte in long-term cutaneous recordings}

Cholinium ion gels were incorporated onto electrodes made of gold and PEDOT:PSS conducting polymer. The electrodes were made on a thin polyimide film allowing a stable and flexible contact to the skin. The cholinium-based ion gel is then used as the electrolytic interface between the skin and the electrode itself. This interface is an important element in successful transcutaneous signal transduction. Fig. 5 illustrates the cross section of the electrode. Polyimide layer allows an easy manipulation of the electrode and its flexibility reduces mechanical stress in wearable conditions (motion, long-term contact...).

The gold layer offers a good electronic conductivity when PEDOT:PSS layer serves to reduce the electrode contact impedance. Additionally, the PEDOT:PSS layer provides a bifunctional ground to monolithically host ion gel due to its conductive and polymeric nature.

In order to compare the performance of these electrodes in cutaneous recordings, we tested the electrode/skin impedance on a healthy volunteer. The electrodes were placed on the subject's arm using a three-electrode configuration, where both counter and reference electrodes were commercial $\mathrm{Ag} / \mathrm{AgCl}$ electrodes. Fig. 6 shows typical electrical impedance spectra, both magnitude and phase angle, for the electrodes tested including different ion gels with different free IL contents. To record physiological potential variations, a good contact with the skin and low impedance are important to ensure a good ionic/electronic translation. All the tested cholinium ion gels showed good long-term adhesion to the skin. This is an important factor since in long-term recordings the electrodes have to endure some mechanical constraints during movements such

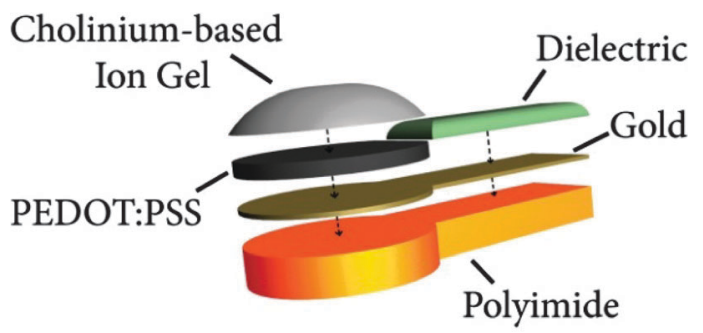

Fig. 5 Exploded view of the different layers of the cutaneous electrode. 


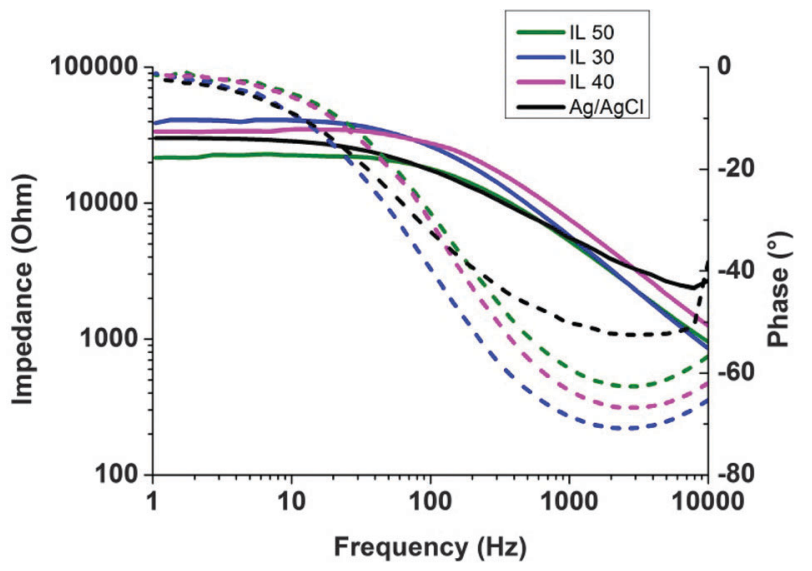

Fig. 6 Impedance values on skin over frequencies for different ion gels and comparison with a standard medical $\mathrm{Ag} / \mathrm{AgCl}$ electrode.

as friction and delamination from the skin. Interestingly, impedance values obtained using electrodes containing different cholinium ion gel compositions are comparable to the ones from regular medical electrode, as shown in Fig. 6. Similar to the data presented above the impedance measurements of the ion gels coated electrodes containing different amounts of the free IL displayed a slight decrease of impedance magnitude at low frequency range with increasing the free IL amount. The phase curves show that ion gel electrodes behave similarly to standard $\mathrm{Ag} /$ $\mathrm{AgCl}$ electrodes, i.e. their phase shift is close to $0^{\circ}$ at low frequencies, as mostly non-polarizable electrodes. The comparison of impedance values with the commercial $\mathrm{Ag} / \mathrm{AgCl}$ electrode standard indicated that cholinium ion gels can effectively be used in electrophysiological recordings. Although impedance of all gels was comparable to the commercial $\mathrm{Ag} / \mathrm{AgCl}$ electrode, the lowest impedance was obtained with IG-50 ion gel formulation. This composition presented a good compromise between mechanical properties and ionic conductivity of the material. It is worth to mention that the ion gels are composed of water soluble ingredients. Therefore, direct exposure of the ion gels to water or excessive sweat will result in loss of gel integrity. However, the exposure of the ion gels to environmental humidity does not affect their integrity. The \% weight gain due to moisture uptake was limited up to $4 \%$ and did not cause any integrity issues of the gels during the evaluated time period, as shown in Fig. S5 (ESI $\dagger$ ). The water uptake from the environment was more important for the ion gels containing higher amount of free ionic liquid. Additionally, the ECG signals recorded during 72 hours did not display any degradation of the signals (Fig. 7) indicating that the integrity of the ion gel was well preserved upon the exposure to environmental and skin humidity.

Different clinical applications cardiac monitoring, nightlong brain activity recordings, prosthesis controlled by muscle activities - require electrodes that can provide a good signal quality for a long period of recording. Most of the current medical electrodes are made with an electrolyte that can dry after a few hours, contrary to ion gels. Dry electrodes were developed in the past decades to overcome limitations related with the gel ambient instabilities. ${ }^{22,23}$ However, they usually
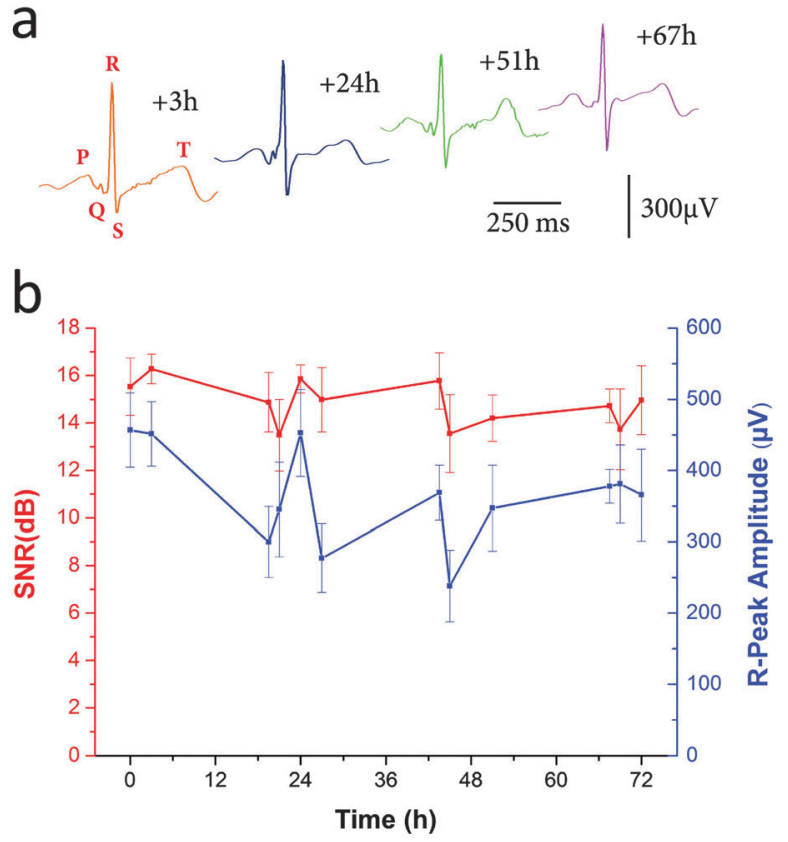

Fig. 7 Long-term ECG measurement performed with IG-50. (A) ECG signals recorded on skin with highlighted PQRST complexes. (B) Stability of the ECG signals over 72 hours with SNR (red) and R-peaks amplitudes (blue) evolutions.

show high sensitivity to motion artefacts due to the limited conformability to the skin. Recently, highly flexible and extremely conformable nanoscale dry electrodes have been developed by Zucca et al. ${ }^{24}$ demonstrating intimate combination of the electrode with skin, repeating its surface topology and showing comparable electrical performance with standard medical ones.

The performance of the cholinium ion gel IG-50 was evaluated in cutaneous electrophysiology. Short-term evaluation of this electrode demonstrated similar performance over $3 \mathrm{~h}$ of recordings in comparison with medical standard, shown in Fig. S6 (ESI $\dagger$ ). To evaluate long-term recording capabilities of IG-50assisted electrodes, the heart activity was regularly recorded from ion gel-assisted electrodes for 3 days long continuously placed in contact with the skin. Electrocardiography is a very common method of monitoring the cardiac function through the electric fields generated by muscular tissues. The progression of cardiac cells depolarization and consecutive muscle contraction throughout the heart give rise to ionic currents that characterize its activity. These small cyclic potential changes are recorded on the skin from electrodes and the signal can be then processed to analyze the different steps of each heart contraction.

ECG signals recorded from our electrodes are shown in Fig. 7a. The PQRST complexes, corresponding to the different depolarization phases of the hearth, can be easily identified. The $\mathrm{R}$ peak matches with the main hearth beat and is used to calculate hearth rate in healthcare applications for general public. Cardiologists need to study the intervals between the different waves as well as general features to be able to diagnose disorders as ventricular hypertrophy or myocardial infarction. Fig. 7b shows the evolution of the ECG signal with time. For 
every measurement Signal-to-Noise Ratio (SNR) was calculated by using the Crest factor, or Peak-to-RMS value, which is the ratio between the $\mathrm{R}$ peak amplitude and the RMS value of the signal all along a PQRST complex. ${ }^{25}$ The amplitudes of R-peaks over time are also shown. As the recordings were carried out on a person performing regular activities (walking, office working, taking shower...) many reasons cause daily variations of SNR and R-peaks amplitudes. The physiological change of the skin impedance, the noise from different electronic sources can interfere with the signal and modify the recording quality. However, the electrodes containing cholinium ion gel allowed obtaining good quality electrophysiological recordings during 72 hours long with the reliable detection of the PQRST cardiac complex.

\section{Experimental}

\section{Materials}

2-Dimethylaminoethyl methacrylate (98\%, Aldrich), 2-bromoethanol (95\%, Aldrich), cholinium hydroxide ( $80 \%$ by weight in methanol, Aldrich), lactic acid (85\% solution in $\mathrm{H}_{2} \mathrm{O}$, Aldrich), 2-hydroxy-2methyl propiophenone (97\%, Aldrich), ethyl acetate (99.5\%, Aldrich), methanol (99\%, Aldrich) were all reagent grade and used without further purification.

\section{Preparation of cholinium ion gels}

Synthesis of the 2-cholinium lactate methacrylate monomer and cholinium lactate IL was explained in detail elsewhere. ${ }^{21,26}$ In order to prepare ion gels containing different amounts of free ILs, calculated amounts of IL, IL monomer and ethylene glycol dimethacrylate crosslinker were mixed in a vial. Appropriate amount $(1.5 \%$ by weight wrt monomer) of photoinitiator was introduced before exposure to UV light. Dymax UVC-5 conveyor belt system with $800 \mathrm{~mW} \mathrm{~cm} \mathrm{~cm}^{-2}$ intensity operating at a wavelength of $365 \mathrm{~nm}$ was used for photopolymerization. Sample to lamp distance was $10 \mathrm{~mm}$ and the belt speed was fixed at $1 \mathrm{~m} \mathrm{~min}^{-1}$. 5 repetitive cycles were applied to achieve a full photopolymerization. The mixture was casted into a mold and photopolymerized. The resulting ion gels were gently removed from the molds and characterized.

\section{Characterization of the cholinium ion gels}

The ${ }^{1} \mathrm{H}$ NMR measurements were carried out on a Bruker AC-400 instrument with the following experimental conditions: spectral width $20 \mathrm{ppm}$ with $32 \mathrm{~K}$ data points, flip angle 90, relaxation delay of $18 \mathrm{~s}$, digital resolution of $0.24 \mathrm{~Hz} / \mathrm{pt}$. Thermogravimetric analysis (TGA) was performed by using a TA instruments Q500 device. The samples were heated from room temperature to $800{ }^{\circ} \mathrm{C}$ with a heating rate of $10{ }^{\circ} \mathrm{C} \mathrm{min} \mathrm{m}^{-1}$ under air. Differential scanning calorimetry (DSC) experiments were performed on a TA Instruments Q2000. The sample materials were enclosed in non-recyclable aluminium hermetic pans, the sample masses being in the range of 5-10 $\mathrm{mg}$. The samples were first heated to $150{ }^{\circ} \mathrm{C}$ with a heating rate of $10{ }^{\circ} \mathrm{C} \min ^{-1}$ and kept isothermal for 5 minutes. The samples were then cooled down to $-80{ }^{\circ} \mathrm{C}$ with a cooling rate of $10{ }^{\circ} \mathrm{C} \mathrm{min}^{-1}$ and kept isothermal for 5 minutes. Second run heating cycles were taken and analyzed for the detection of the glass transition temperature of the ion gels. Rheology measurements on the prepared ion gels were conducted on a Thermo-Haake Rheostress I viscoelastometer using oscillatory tests. Angular frequency sweeps from 0.01 to $100 \mathrm{rad} \mathrm{s}^{-1}$ at constant strain amplitude $(\gamma=0.005)$ were applied at $25{ }^{\circ} \mathrm{C} . G^{\prime}$ and $G^{\prime \prime}$ values were plotted versus angular frequency. Attenuated Total Reflection Fourier Transform Infrared Spectroscopy (ATR-FTIR) was performed to confirm the success of the photopolymerization. Spectra were recorded on Bruker ALPHA-P equipment from $350 \mathrm{~cm}^{-1}$ to $4000 \mathrm{~cm}^{-1}$ with a resolution of $2 \mathrm{~cm}^{-1}$. Ionic conductivities $(\sigma)$ of ion gels at different temperatures were obtained by Electrochemical Impedance Spectroscopy (EIS) using a Bio-logic VMP3 multichannel potentiostaticgalvanostatic system equipped with an impedance module. The experiment was performed using a 2-electrode Swagelok ${ }^{\circledR}$ cell in which a coin-like shape membrane was sandwiched in between two stainless steel pistons. Samples were exposed to different temperature in an environmental chamber (BINDER, Model MK 53 (E2)) interfaced with APTCOM3 software. Each temperature was stabilized for at least $1 \mathrm{~h}$ before each measurement. The frequency range was varied from $1 \mathrm{MHz}$ to $10 \mathrm{MHz}$ at the bias voltage of $0 \mathrm{~V}$ with a potential amplitude of $10 \mathrm{mV}$. Ionic conductivity $(\sigma)$ for each temperature was calculated using the following expression; $\sigma\left(\mathrm{S} \mathrm{cm}^{-1}\right)=t / R \times A$, where, $t$ is the thickness of the membrane (cm), $A$ is geometrical area of the membrane $\left(\mathrm{cm}^{2}\right)$ and $R$ is the resistance calculated by the intercept of the curve with the real axis on a Nyquist plot $(\Omega)$.

\section{Preparation of electrodes and ECG measurements}

Electrodes were made according to the previously developed protocol described by Leleux et al. ${ }^{27}$ Briefly, we used a laser-cut plastic polyimide (Kapton $\mathrm{HN}$ ) electrodes with a thickness of $125 \mu \mathrm{m}$ and an active area of $0.5 \mathrm{~cm}$ square. $10 \mathrm{~nm}$ of chromium and $100 \mathrm{~nm}$ of gold were evaporated on the Kapton electrode. The electrode active area was then coated by drop casting with $5 \mu \mathrm{L}$ of a solution of poly(3,4-ethylenedioxythiophene) doped with poly(styrene sulfonate) (PEDOT:PSS) from (Clevios PH-1000 from Heraeus Holding GmbH). PEDOT:PSS was mixed with ethylene glycol (from Sigma-Aldrich), 4-dodecylbenzenesulfonic acid (Sigma Aldrich), and 3-methacryloxypropyltrimethoxysilane (Sigma Aldrich) with a ratio of $8 / 2 / 0.004 / 0.1$ respectively to improve both conductivity, surface energy and surface adhesion. $15 \mu \mathrm{L}$ of cholinium ion gel solution mixed with the photoinitiator were deposited on top of the PEDOT:PSS layer of $0.5 \mathrm{~cm}^{2}$ (size of the recording area) and UV cured until the full polymerization, as described above. The resulting layer of ion gel was around $2 \mathrm{~mm}$ thick. The connection was added at the other end of the electrode using a snap button and the insulation was made using a protective paint.

The electrodes were characterized accordingly to the ANSI/ AAMI EC12:2000/(R)2005 norm: AC impedance and DC offset voltage are $300 \Omega$ and $25.4 \mathrm{mV}$, respectively. The skin impedance spectra were acquired using a potentiostat (Autolab equipped with FRA module, Metrohm B.V.), using a 3 electrodes 
configuration. Two electrodes, working and counter, were placed $2 \mathrm{~cm}$ apart from each other on the forearm and a third one, called reference electrode, was located $30 \mathrm{~cm}$ away. ${ }^{23}$

ECG recordings were performed by placing one electrode on each wrist of a healthy volunteer to form a bipolar Limb Lead I derivation, one of the 12 ECG lead configurations used in clinics. ${ }^{28,29}$ To avoid any loose of contact due to the possible mechanical displacement of the electrode connected to electrical wires during long-term evaluations, the electrodes were fixed to the skin with $3 \mathrm{M}$ Steri-Strip and a protective bandage was rolled around each wrist. The ECG was then recorded for 48 seconds several times a day during 72 hours. The CardioTOMM acquisition system was provided by Microvitae Technologies. Signals were then filtered and processed by LabVIEW software using a standard wavelet approach.

\section{Conclusions}

New cholinium-based ion gels were synthesized by photopolymerization in less than two minutes. The ion gels showed mechanical properties of an elastic gel soft solid and low glass transition temperatures and thermal stability. As an important property, the ion gels showed values of ionic conductivity values at $20{ }^{\circ} \mathrm{C}$ up to $10^{-3} \mathrm{~S} \mathrm{~cm}^{-1}$. These properties make the cholinium-based ion gel good candidates to be used as the electrolytic interface with the skin in transcutaneous electrophysiology. Cholinium ion gels showed good long term adhesion to the skin without any loss of properties caused by the evaporation of the electrolyte in commercial electrodes. Accurate physiologic signals were recorded for a long period of time with a remarkable stability. Due to the recognized low toxicity of cholinium ionic liquids, these ion gels are ideal candidates to assist the long-term cutaneous recordings. However, further evaluations are required to assess the dermotoxicology of the ion gels in clinical applications.

\section{Acknowledgements}

The financial support of European Commission through project Renaissance-ITN 289347 is greatly acknowledged.

\section{Notes and references}

1 L.-D. Liao, I.-J. Wang, S.-F. Chen, J.-Y. Chang and C.-T. Lin, Sensors, 2011, 11, 5819.

2 M. Galinski, A. Lewandowsky and I. Stepniak, Electrochim. Acta, 2006, 51, 5567.

3 P. Leleux, C. Johnson, X. Strakosas, J. Rivnay, T. Herve, R. M. Owens and G. G. Malliaras, Adv. Healthcare Mater., 2014, 3, 1377.

4 T. P. T. Pham, C.-W. Cho and Y.-S. Yun, Water Res., 2010, 44, 352.

5 S. Stolte, J. Arning, U. Bottin-Weber, A. Muller, W.-R. Pitner, U. Welz-Biermann, B. Jastorff and J. Ranke, Green Chem., 2007, 9, 760-767; S. Stolte, M. Matzke, J. Arning, A. Boschen,
W.-R. Pitner, U. Welz-Biermann, B. Jastorff and J. Ranke, Green Chem., 2007, 9, 1170-1179.

6 S. Stolte, J. Arning, U. Bottin-Weber, M. Matzke, F. Stock, K. Thiele, M. Uerdingen, U. Welz-Biermann, B. Jastorff and J. Ranke, Green Chem., 2006, 8, 621.

7 A. Romero, A. Santos, J. Tojo and A. Rodriguez, J. Hazard. Mater., 2008, 151, 268.

8 A. Cieniecka-Rosłonkiewicz, J. Pernak, J. Kubis-Feder, A. Ramani, A. J. Robertson and K. R. Seddon, Green Chem., 2005, 7, 855.

9 A. Garcia-Lorenzo, E. Tojo, J. Tojo, M. Teijeira, F. J. RodríguezBerrocal, M. P. González and V. S. Martínez-Zorzan, Green Chem., 2008, 10, 508.

10 M. Petkovic, J. L. Ferguson, H. Q. N. Gunaratne, R. Ferreira, K. R. Seddon, L. P. N. Rebelo and C. S. Pereira, Green Chem., 2010, 12, 643.

11 Y. Fukaya, Y. Iizuka, K. Sekikawa and H. Ohno, Green Chem., 2007, 9, 1155.

12 T. P. Lodge, Science, 2008, 321, 50; L. D. McIntosh, T. Kubo and T. P. Lodge, Macromolecules, 2014, 47, 1090-1098.

13 J. L. Bideau, L. Viau and A. Vioux, Chem. Soc. Rev., 2011, 40, 907. 14 (a) G. A. Tiruye, D. Muñoz-Torrero, J. Palma, M. Andersson and R. Marcilla, J. Power Sources, 2015, 279, 472; (b) A. S. Shaplov, R. Marcilla and D. Mecerreyes, Electrochim. Acta, 2015, DOI: 10.1016/j.electacta.2015.03.038.

15 M. Diaz, A. Ortiz, M. Isik, D. Mecerreyes and I. Ortiz, Int. J. Hydrogen Energy, 2015, DOI: 10.1016/j.ijhydene.2015.03.109.

16 D. Kodagholy, V. F. Curto, K. J. Fraser, M. Gurfinkel, R. Byrne, D. Diamond, G. G. Malliaras, F. Benito-Lopez and R. M. Owens, J. Mater. Chem., 2012, 22, 4440.

17 S. Imauzumi, H. Kokubo and M. Watanabe, Macromolecules, 2012, 45, 401.

18 C. Muller, M. Hamedi, R. Karlsson, R. Jansson, R. Marcilla, M. Hedhammar and O. Inganas, Adv. Mater., 2011, 23, 898-901.

19 J.-H. Choi, W. Xie, Y. Gu, C. D. Frisbie and T. P. Lodge, ACS Appl. Mater. Interfaces, 2015, 7, 7294-7302.

20 L. Viau, C. Tourne-Peteilh, J.-M. Devoisselle and A. Vioux, Chem. Commun., 2010, 46, 228.

21 M. Isik, R. Garcia, L. C. Kollnus, L. C. Tome, I. M. Marrucho and D. Mecerreyes, ACS Macro Lett., 2013, 2, 975.

22 A. Searle and L. Kirkup, Physiol. Meas., 2000, 21, 271-283.

23 N. Meziane, J. G. Webster, M. Attari and A. J. Nimunkar, Physiol. Meas., 2013, 34, R47-R69.

24 A. Zucca, C. Cipriani, S. Sudha, S. Tarantino, D. Ricci, V. Mattoli and F. Greco, Adv. Healthcare Mater., 2015, 4, 941.

25 G. Clifford, F. Azuaje and P. McSharry, Advanced Methods and Tools for ECG Analysis, Artech House Publishing, Boston, London, 2006, p. 384.

26 M. Isik, H. Sardon, M. Sanchez and D. Mecerreyes, RSC Adv., 2014, 4, 53407.

27 P. Leleux, J.-M. Badier, J. Rivnay, C. Bénar, T. Hervé, P. Chauvel and G. G. Malliaras, Adv. Healthcare Mater., 2013, 3, 490-493.

28 W. Einthoven, G. Fahr and A. de Waart, Pflüger Arch. ges. Physiol., 2013, 150, 275-315.

29 A. R. Barens, F. N. Wilson, H. E. B. Pardee, C. C. Wolpferth and P. D. White, Am. Heart J., 1938, 15, 235-239. 